\title{
Erratum to: ISOexpresso: a web-based platform for isoform-level expression analysis in human cancer
}

\author{
In Seok Yang ${ }^{1}$, Hyeonju Son ${ }^{1,2}$, Sora Kim ${ }^{1,2}$ and Sangwoo Kim ${ }^{1,2^{*}}$
}

\section{Erratum}

In the original publication of this article [1], the grant number HI15C1234 is incorrect and should be corrected to HI15C1601.

The correction details of the grant number can be found below:

\section{Funding}

This research was supported by a grant of the Korea Health Technology R\&D Project through the Korea Health Industry Development Institute (KHIDI), funded by the Ministry of Health \& Welfare, Republic of Korea (grant number : H115C1601).

Received: 11 October 2016 Accepted: 20 October 2016

Published online: 25 October 2016

\section{Reference}

1. Yang IS, et al. ISOexpresso: a web-based platform for isoform-level expression analysis in human cancer. BMC Genomics. 2016;17:631. doi:10.1186/s12864-016-2852-6.

\footnotetext{
* Correspondence: swkim@yuhs.ac

'Severance Biomedical Science Institute, Yonsei University College of Medicine, 50-1 Yonsei-ro, Seoul 03722, Korea

${ }^{2}$ Brain Korea 21 PLUS Project for Medical Sciences, Yonsei University College of Medicine, 50-1 Yonsei-ro, Seoul 03722, Korea
} 